\title{
Relationship between rainfall, runoff, soil loss and productivity in north eastern ghat zone of odisha
}

\begin{abstract}
A trial was conducted at All India Coordinated Research Project ,OUAT, Phulbani during the year 2007-09 with the objectives to quantify the runoff and soil loss under different cropping systems and develop relationship among them. The treatments tried were $T_{1}$-Sole crop of rice, $T_{2}$ - Sole crop of pigeon pea. $T_{3}$-Sole crop of groundnut, $T_{4}$ Pigeonpea and rice in alternate strips, $\mathrm{T}_{5}$ - Pigeon pea and groundnut in alternate strips, $\mathrm{T}_{6}$-Intercrop of rice and pigeon pea $(5: 2), \mathrm{T}_{7}$ - Intercrop of groundnut and pigeon pea (4:2), $\mathrm{T}_{8}$-Uncultivated fallow, $\mathrm{T}_{9}$-Cultivated fallow, All crops were planted across the contour. Intercrop of groundnut and pigeon pea (4:2) gave significantly higher rice equivalent yield compared to other sole crops. Mean rice equivalent yield was $38.62 \mathrm{q} /$ ha. Groundnut + pigeon pea (4:2) introduction increased the yield by $158 \%$ as $97 \%$ and $21 \%$ when compared with sole crop of rice, pigeon pea and groundnut respectively. Groundnut + pigeon pea $(4: 2)$ gave the lowest runoff of $309 \mathrm{~mm}$ which is $23 \%$ less than the cultivated fallow $(401 \mathrm{~mm})$. Groundnut + pigeon pea $(4: 2)$ gave the lowest soil loss $(8.03 \mathrm{t} / \mathrm{ha})$ which is $47 \%$ lower than the cultivated fallow (with highest soil loss $15.19 \mathrm{t} / \mathrm{ha})$. The Groundnut + pigeon pea $(4: 2)$ gave the lowest $(24.2 \%)$ mean runoff of the rainfall compared to other treatments. The relationship among rainfall, runoff and soil loss was found out which can be used to predict the runoff and soil loss from rainfall for same type of soil condition and slope. The Thus it can be concluded that intercropping of groundnut with pigeon pea planted along contour may be practiced to increase crop yield and lowering the soil loss and runoff in the hilly tribal areas of Kandhamal district of Odisha.
\end{abstract}

Keywords: relationship, rainfall, runoff, soil loss, productivity
Volume 4 Issue 2 - 2016

\author{
Subudhi CR,' Senapati SC ${ }^{2}$ \\ 'Associate Professor, Department of SWCE, Orissa University \\ of Agriculture and Technology, India \\ ${ }^{2}$ Professor, Department of SWCE, Orissa University of \\ Agriculture and Technology, India
}

Correspondence: Subudhi CR, Associate Professor (SWCE), CAET, OUAT, BBSR-3, College of Agricultural Engineering and Technology, Orissa University of Agriculture and Technology, Bhubaneswar, 751003, India, Email rsubudhi5906@gmail.com

Received: June 20, 2016 | Published: July 22, 2016

\section{Introduction}

Strip and intercropping of cereal crops with pulses/oilseeds are approved practices of breaking long slopes, which prevent soil loss, reduce runoff and enhance productivity. Therefore, this experiment has been designed to know the effect of strip as well as intercrop of pigeonpea, rice and groundnut on runoff, soil loss and productivity on sloppy agricultural land. These intangible benefits have not been assessed properly. Hence the present experiment has been designed. Samra JS ${ }^{1}$ reported that renovation of terrace and plantation of fruit plants, timber plants improved biomass production, net returns, growth of crop, productivity, reduction of runoff in the range of 1.510.8 times, peak flow rate by 20 times\& soil loss in the range of 1.2 to 5.2 times ,as well as water table rise. Subudhi et al., ${ }^{2}$ have reported that effect of vegetative barrier like Vetiver has increased the rice yield, decrease the soil loss and decrease the runoff compared to farmers practice. Arora et al., ${ }^{3}$ reported that there is a growing need for rain water management since $96 \mathrm{~m}$ ha out of $142 \mathrm{~m}$ ha of net cultivated land of the country is rainfed. Scientific use of these resources will definitely increase the productivity \&conservation of resources like soil \& water. Kumar ${ }^{4}$ reported that impact of different soil\& water conservation techniques viz. contour bunding, terracing, land leveling, smoothening\& gully plugging, sowing across the slope, vegetative barrier, increase the Kharif crops by 25-30 percent. Establishment of vegetative barrier with mechanical measures were more effective in controlling soil erosion $\left(3.8 \mathrm{tha}^{-1}\right)$ over conventional method(9.64 $\mathrm{t} \mathrm{ha}^{-1}$ )and runoff thereby making more moisture available for crop growth. Anonymous ${ }^{5-7}$ reported that intercropping of groundnut with pigeonpea planted along contour gave the highest rice equivalent yield, lowest soil loss and runoff.

\section{Objectives}

To quantify the runoff and soil loss under different cropping systems and develop relationship among them.

\section{Materials and methods}

A trial was conducted at All India Coordinated Research Project, OUAT, Phulbani during the year 2007-09. The experiment was laid out on $2 \%$ land slope. Multi slot division box and drums were put to measure the runoff and soil loss daily after each rainfall. The treatments tried were $\mathrm{T}_{1}$-Sole crop of rice, $\mathrm{T}_{2}$ - Sole crop of pigeon pea. $\mathrm{T}_{3}$-Sole crop of groundnut, $\mathrm{T}_{4}$-Pigeonpea and rice in alternate strips, $\mathrm{T}_{5}$ - Pigeon pea and groundnut in alternate strips, $\mathrm{T}_{6}$-Intercrop of rice and pigeon pea (5:2), $\mathrm{T}_{7}$ - Intercrop of groundnut and pigeon pea (4:2), $\mathrm{T}_{8}$-Uncultivated fallow, $\mathrm{T}_{9}$-Cultivated fallow, All crops were planted across the contour. Different crops and their varieties are, Rice- ZHU11-26; Pigeon pea - UPAS-120; Groundnut- Smruti (OG-52-1). The experiment design was Randomized Block Design and numbers of replications were three. Plot size was $25 \mathrm{mX} 2 \mathrm{~m}$. Seed rate were, Rice$75 \mathrm{~kg} / \mathrm{ha}$; Pigeon pea- $25 \mathrm{~kg} / \mathrm{ha}$; Groundnut- $150 \mathrm{~kg} / \mathrm{ha}$ (pod). Fertilizer applied was;

Basal: Rice-30:30:30kg N-P $\mathrm{O}_{5}-\mathrm{K}_{2} \mathrm{O} / \mathrm{ha}$; Pigeon pea-20:40:20kg $\mathrm{N}-\mathrm{P}_{2} \mathrm{O}_{5}-\mathrm{K}_{2} \mathrm{O} /$ haGroundnut-20: 40:40 kg N-P $\mathrm{O}_{5}-\mathrm{K}_{2} \mathrm{O} /$ ha.

\section{Top dressing: Rice- $30 \mathrm{~kg} \mathbf{N}$ in two splits.}

The runoff collected daily at $8 \mathrm{AM}$ was measured from the drum and 1 lit of runoff from each drum were collected for silt analysis, so soil loss can be measured from the silt sample collected after evaporating the sample in the heater. The rainfall was also measured. Thus the relation between rainfall $(\mathrm{mm})$-runoff $(\mathrm{mm})$, rainfall $(\mathrm{mm})$ - 
soil loss (t/ha) and runoff (mm)-soil loss (t/ha) were calculated and coefficient of determination was also calculated.

\section{Result and discussion}

Rainfall, runoff and soil loss: During 2009 Pigeon pea received the highest amount of rainfall $(1544.8 \mathrm{~mm})$ this cropping treatment received the highest amount of runoff $(516 \mathrm{~mm})$ In addition, Groundnut
+ pigeon pea (4:2) gave the lowest runoff of $309 \mathrm{~mm}$ which is $23 \%$ less than the cultivated fallow $(401 \mathrm{~mm})$. Groundnut + pigeon pea $(4: 2)$ gave the lowest soil loss $(8.03 \mathrm{t} / \mathrm{ha}$ ) (Table 1) which is $47 \%$ lower than the cultivated fallow (with highest soil loss $15.19 \mathrm{t} / \mathrm{ha}$ ). The Groundnut + pigeon pea $(4: 2)$ gave the lowest $(24.2 \%)$ mean runoff of the rainfall compared to other treatments. The poor and marginal farmers having lands in upland ecosystem may go for this technology Figures 1-6.

Table I Runoff and soil loss under different cropping systems during 2007 to 2009 (3 years)

\begin{tabular}{|c|c|c|c|c|c|c|c|c|c|c|c|c|}
\hline \multirow{2}{*}{ Treatments } & \multicolumn{4}{|c|}{ Run off (mm) } & \multicolumn{4}{|c|}{ Soil loss(t/ha) } & \multicolumn{4}{|c|}{ Run off (\% of rainfall) } \\
\hline & 2007 & 8 & 9 & Mean & 2007 & 8 & 9 & Mean & 2007 & 2008 & 2009 & Mean \\
\hline TI - Rc Sole & 221.268 & 295.34 & 514 & 344 & 6.72 & 10.01 & 12.6 & 9.78 & 21.7 & 25.3 & 33.3 & 26.8 \\
\hline T2- Pp Sole & 226.92 & 296.43 & 516 & 347 & 7.116 & 10.06 & 12.65 & 9.94 & 22.26 & 25.4 & 33.4 & 27 \\
\hline T3-Gn Sole & 221.943 & 284.03 & 508 & 338 & 6.49 & 9.35 & 12.43 & 9.42 & 21.77 & 24.3 & 32.9 & 26.3 \\
\hline $\begin{array}{l}\text { T4-Pp \& Rc strip } \\
\text { cropping }\end{array}$ & 221.1 & 279.48 & 487 & 329 & 6.47 & 9.59 & 11.84 & 9.3 & 21.68 & 23.9 & 31.5 & 25.7 \\
\hline $\begin{array}{l}\text { T6-Rc + Pp }(5: 2) \\
\text { intercropping }\end{array}$ & 217.725 & 270.03 & 471 & 320 & 6.358 & 9.32 & 9.81 & 8.5 & 21.35 & 23.1 & 30.5 & 25 \\
\hline $\begin{array}{l}\text { T7-Gn+ Pp (4:2) } \\
\text { intercropping }\end{array}$ & 213.376 & 258.39 & 456 & 309 & 6.231 & 8.61 & 9.25 & 8.03 & 20.96 & 22.1 & 29.5 & 24.2 \\
\hline $\begin{array}{l}\text { T8- Uncultivated } \\
\text { fallow }\end{array}$ & 234.175 & 347.72 & 555 & 379 & 7.395 & 11.92 & 17.84 & 12.39 & 22.97 & 29.8 & 35.9 & 29.6 \\
\hline $\begin{array}{l}\text { T9- Cultivated } \\
\text { fallow }\end{array}$ & 250.119 & 364.68 & 588 & 401 & 9.772 & 15.99 & 19.82 & 15.19 & 24.53 & 31.2 & 38.1 & 31.3 \\
\hline Mean & 225.214 & 296.88 & 509 & 344 & 7.029 & 10.48 & 13.11 & 10.21 & 22.09 & 25.4 & 32.9 & 26.8 \\
\hline
\end{tabular}

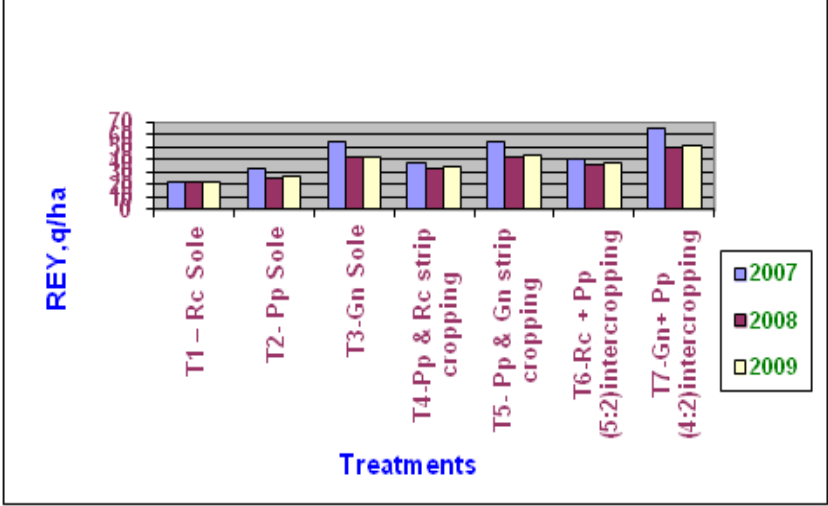

Figure I Rice equivalent yield in different years.

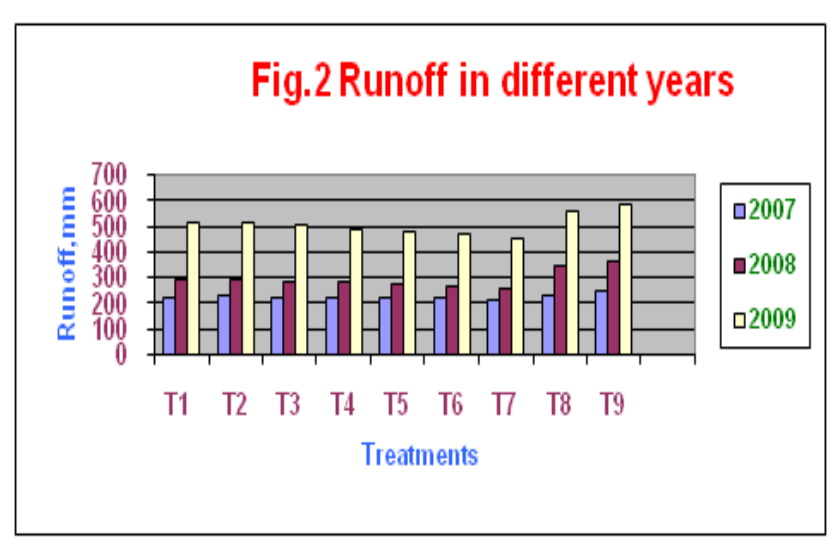

Figure 2 Runoff in different year. 


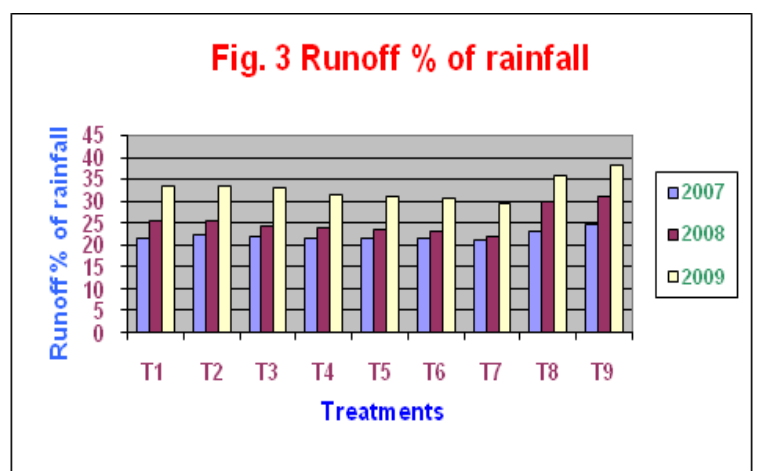

Figure 3 Runoff \% of rainfall.

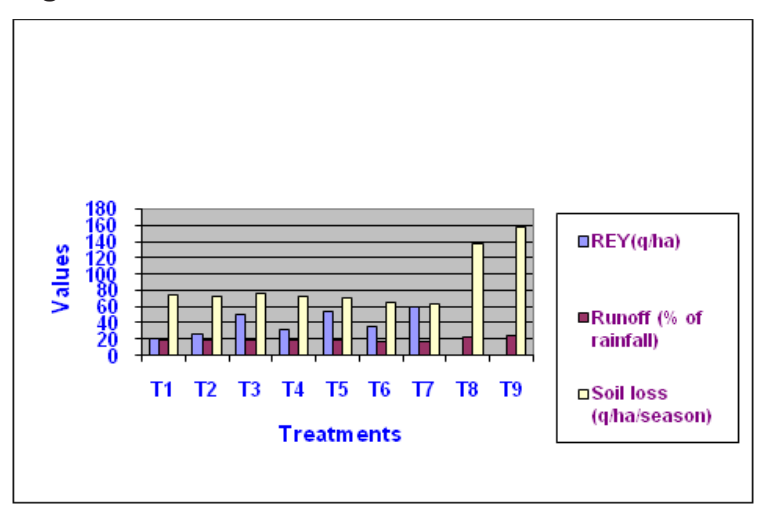

Figure 5 Rice Equivalent Yield (REY), Runoff and soil loss in different treatments.
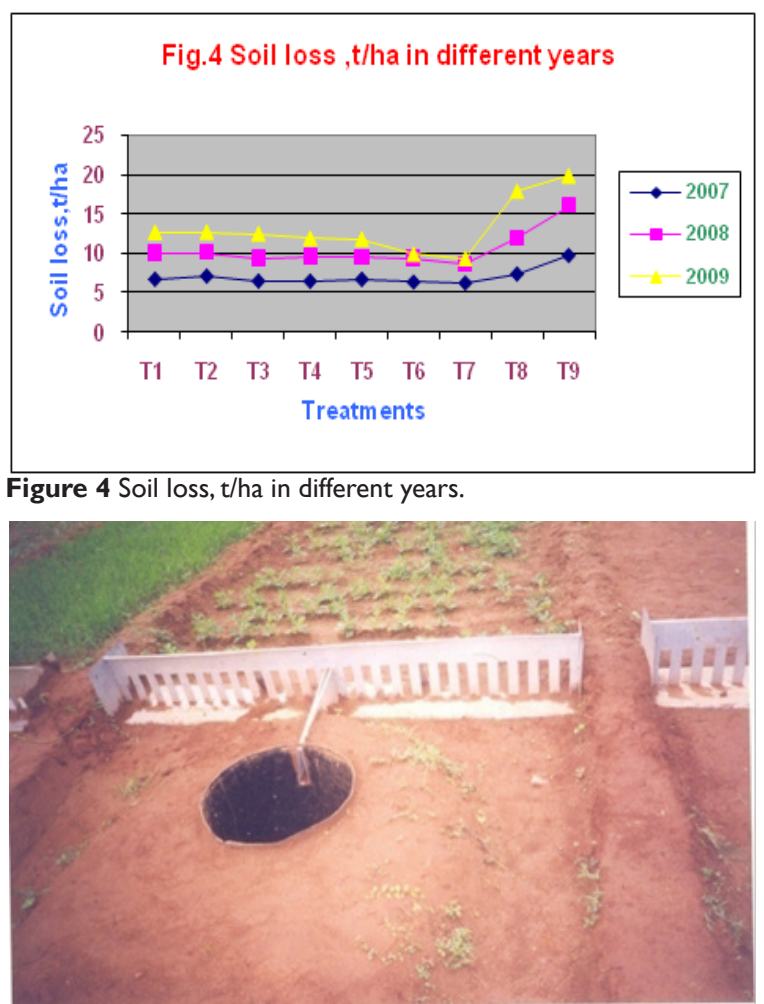

Figure 6 Multi slot division box with runoff collection tank.
Moisture content: From Table 2 it is observed that the Gn+Pp (4:2) intercropping gave highest moisture content, plant height and other yield attributing characters compared to other treatments so moisture content might be the reason to increase the yield.

Table 2 Mean moisture content and yield attributing characteristics during 2007-09

\begin{tabular}{ll}
\hline & Mean \\
\cline { 2 - 2 } Treatments & Moisture \\
\cline { 2 - 2 } & Content \\
\hline T1 - Rc Sole & 15.5 \\
T2- Pp Sole & 16.1 \\
T3-Gn Sole & 16 \\
T4-Pp \& Rc strip cropping & 16.2 \\
T5- Pp \& Gn strip cropping & 17.6 \\
T6-Rc + Pp (5:2)intercropping & 17.1 \\
T7-Gn+ Pp (4:2)intercropping & 17.8 \\
T8- Uncultivated fallow & 15.6 \\
T9- Cultivated fallow & 15.2 \\
SE (m)+ & 0.251 \\
CD $(0.05)$ & 0.903 \\
Mean & 16.3 \\
\hline
\end{tabular}

Nutrient loss: Nutrient loss in different treatments were shown in Table 3, it is observed that total nutrient loss was highest in treatment 9 i.e. Cultivated fallow may be due to the reason that soil surface was exposed without any crop and cultivated and also soil loss was more in this treatment compared to other treatments. Lowest nutrient loss was in $\mathrm{T}_{7}(42.21 \mathrm{~kg} / \mathrm{ha})$ i.e. - $\mathrm{Gn}+\mathrm{Pp}$ (4:2) intercropping.

Table 3 Nutrient lost from soil sample collected from runoff from different treatments

\begin{tabular}{lllll}
\hline & \multicolumn{3}{l}{$\begin{array}{l}\text { Nutrient loss from } \\
\text { different treatments, } \\
\text { Kg/ha }\end{array}$} & $\begin{array}{l}\text { Total } \\
\text { nutrient } \\
\text { loss, kg/ha }\end{array}$ \\
\cline { 2 - 5 } & $\mathbf{N}$ & $\mathbf{P}_{2}$ O5 & $\mathbf{K}_{2}$ O & \\
\hline TI - Rc Sole & 11.5 & 10.14 & 29.7 & 51.34 \\
$\begin{array}{l}\text { T2- Pp Sole } \\
\text { T3-Gn Sole }\end{array}$ & 11.5 & 13.24 & 35.62 & 60.36 \\
$\begin{array}{l}\text { T4-Pp \& Rc strip } \\
\text { cropping }\end{array}$ & 13.13 & 12 & 28.76 & 53.89 \\
$\begin{array}{l}\text { T5- Pp \& Gn strip } \\
\text { cropping }\end{array}$ & 11.13 & 9.69 & 23.52 & 44.34 \\
$\begin{array}{l}\text { T6-Rc + Pp (5:2) } \\
\text { intercropping }\end{array}$ & 11.5 & 7.16 & 27.42 & 46.08 \\
$\begin{array}{l}\text { T7-Gn+ Pp (4:2) } \\
\text { intercropping }\end{array}$ & 12.25 & 4.62 & 25.34 & 42.21 \\
$\begin{array}{l}\text { T8- Uncultivated fallow } \\
\begin{array}{l}\text { T9- Cultivated fallow } \\
\hline\end{array}\end{array}$ & 11.5 & 4.17 & 36.02 & 51.69 \\
\hline
\end{tabular}


Yield attributing characteristics: The biometric characteristics of different crops under different treatments is given in Table 4 it is observed that the $\mathrm{Gn}+\mathrm{Pp}$ (4:2) intercropping gave highest plant height and other yield attributing characters compared to other treatments.

Table 4 Mean yield attributing characteristics during 2007-09

\begin{tabular}{|c|c|c|c|c|c|c|c|c|c|}
\hline \multirow[b]{2}{*}{ Treatments } & \multicolumn{3}{|l|}{ Rice } & \multicolumn{3}{|l|}{ Pigeonpea } & \multicolumn{3}{|c|}{ Groundnut } \\
\hline & $\begin{array}{l}\text { Plant } \\
\text { height, } \\
\text { cm }\end{array}$ & $\begin{array}{l}\text { Penicle } \\
\text { length, } \\
\mathrm{cm}\end{array}$ & $\begin{array}{l}\text { No of tillers } \\
\text { /m run }\end{array}$ & $\begin{array}{l}\text { Plant } \\
\text { height, cm }\end{array}$ & $\begin{array}{l}\text { Spread, } \\
\text { cm }\end{array}$ & $\begin{array}{l}\text { No of } \\
\text { branches }\end{array}$ & $\begin{array}{l}\text { Plant } \\
\text { height, } \\
\text { cm }\end{array}$ & $\begin{array}{l}\text { No of } \\
\text { branches }\end{array}$ & $\begin{array}{l}\text { No of } \\
\text { pods per } \\
\text { plant }\end{array}$ \\
\hline TI - Rc Sole & 71.4 & 17 & 51.5 & & & & & & \\
\hline T2- Pp Sole & & & & 186.6 & 93.2 & 22.1 & & & \\
\hline T3-Gn Sole & & & & & & & 65.4 & 8 & 35.2 \\
\hline $\begin{array}{l}\text { T4-Pp \& Rc strip } \\
\text { cropping }\end{array}$ & 74.6 & 17.4 & 53.8 & 191.6 & 99.4 & 22.2 & & & \\
\hline $\begin{array}{l}\text { T5- Pp \& Gn strip } \\
\text { cropping }\end{array}$ & & & & 198 & 106 & 23.1 & 74.5 & 8.6 & 37.9 \\
\hline $\begin{array}{l}\text { T7-Gn+ Pp ( } 4: 2) \\
\text { inter cropping }\end{array}$ & & & & 209.6 & 106.8 & 23.1 & 76 & 9 & 42.1 \\
\hline Mean & 74.7 & 17.5 & 55.5 & 197.5 & 100.7 & 22.5 & 72 & 8.5 & 38.4 \\
\hline
\end{tabular}

Yield: Intercrop of groundnut and pigeonpea (4:2) gave significantly higher rice equivalent yield compared to other sole crops. Mean rice equivalent yield was $38.62 \mathrm{q} / \mathrm{ha}$. Groundnut + pigeonpea $(4: 2)$

Table 5 Rice equivalent yield, under different cropping systems during 2007 to 2009 (3 years)

\begin{tabular}{|c|c|c|c|c|}
\hline \multirow{2}{*}{ Treatments } & \multicolumn{4}{|c|}{$\operatorname{REY}(q / h a)$} \\
\hline & 2007 & 8 & 9 & Mean \\
\hline TI - Rc Sole & 21.047 & 21.17 & 22.38 & 21.53 \\
\hline T2- Pp Sole & 33.393 & 25.436 & 25.85 & 28.23 \\
\hline T3-Gn Sole & 54.179 & 41.307 & 41.87 & 45.79 \\
\hline $\begin{array}{l}\text { T4-Pp \& Rc strip } \\
\text { cropping }\end{array}$ & 37.528 & 32.584 & 33.7 & 34.6 \\
\hline $\begin{array}{l}\text { T5- Pp \& Gn strip } \\
\text { cropping }\end{array}$ & 53.784 & 42.448 & 44.25 & 46.83 \\
\hline $\begin{array}{l}\mathrm{T} 6-\mathrm{Rc}+\mathrm{Pp}(5: 2) \\
\text { intercropping }\end{array}$ & 40.429 & 35.843 & 37.08 & 37.78 \\
\hline $\begin{array}{l}\text { T7-Gn+ Pp }(4: 2) \\
\text { intercropping }\end{array}$ & 65.996 & 49.043 & 51.68 & 55.57 \\
\hline \multicolumn{5}{|c|}{ T8- Uncultivated fallow } \\
\hline \multicolumn{5}{|c|}{ T9- Cultivated fallow } \\
\hline $\mathrm{SE}(\mathrm{m})+$ & 0.961 & 0.612 & 0.601 & 0.586 \\
\hline$C D(0.05)$ & 2.914 & 1.885 & 1.823 & 1.778 \\
\hline Mean & 43.78 & 35.404 & 36.69 & 38.62 \\
\hline
\end{tabular}

introduction increased the yield by $158 \%$ as $97 \%$ and $21 \%$ when compared with sole crop of rice, pigeonpea and groundnut respectively (Table 5).

Economics: From Table 6 it is observed that the Gn+Pp (4:2) intercropping gave highest mean $\mathrm{B}: \mathrm{C}$ ratio i.e. 2.06 compared to all other treatments among all the cropping system treatments.

Table 6 Economics and Rain water use efficiency during 2007-09 as affected by different in-situ conservation practices

\begin{tabular}{llllll}
\hline Treatments & $\begin{array}{l}\text { Cost of } \\
\text { Cultivation } \\
\text { (Rs/ha) }\end{array}$ & $\begin{array}{l}\text { Gross } \\
\text { Income } \\
\text { (Rs/ha) }\end{array}$ & $\begin{array}{l}\text { Net } \\
\text { Income } \\
\text { (Rs/ha) }\end{array}$ & $\begin{array}{l}\text { B:C } \\
\text { Ratio }\end{array}$ & $\begin{array}{l}\text { Rain } \\
\text { Water } \\
\text { Ufficiency } \\
\text { (Kg/ha/ } \\
\text { mm) }\end{array}$ \\
\hline TI-Rc Sole & 14,000 & 17,933 & 3,933 & 1.28 & 1.62 \\
T2- Pp Sole & 14,200 & 23,391 & 9,191 & 1.65 & 1.67 \\
T3-Gn Sole & 21,721 & 39,582 & 17,861 & 1.82 & 2.91 \\
$\begin{array}{l}\text { T4-Pp \& Rc } \\
\text { strip cropping }\end{array}$ & 17,861 & 28,866 & 11,005 & 1.62 & 2.18
\end{tabular}

\begin{tabular}{lccccc}
$\begin{array}{l}\text { T5- Pp \& Gn } \\
\text { strip cropping }\end{array}$ & 19,294 & 38,839 & 19,545 & 2.01 & 2.86 \\
$\begin{array}{l}\text { T6-Rc }+ \\
\begin{array}{l}\text { Pp (5:2) } \\
\text { intercropping }\end{array}\end{array}$ & 19,166 & 32,222 & 13,056 & 1.68 & 2.4 \\
$\begin{array}{l}\text { T7-Gn+ } \\
\begin{array}{l}\text { Pp (4:2) } \\
\text { intercropping }\end{array}\end{array}$ & 22,577 & 46,411 & 23,834 & 2.06 & 3.35 \\
\hline
\end{tabular}


The relationship between rainfall ( $\mathrm{mm})$, runoff $(\mathrm{mm})$ and soil loss t/ha was presented in Table 7-9. The correlation coefficient was also found out. The relationship among rainfall, runoff and soil loss was found out which can be used to predict the runoff and soil loss from rainfall for same type of soil condition and slope.

Table 7 Relation between Rainfall (X)mm, Runoff(Y)mm and Soil loss (Z) t/ha in different treatments along with co-efficient of determination (2007-08)

\begin{tabular}{|c|c|c|c|}
\hline \multirow[b]{2}{*}{ Treatments } & \multicolumn{3}{|l|}{ Relations } \\
\hline & $\begin{array}{l}\text { Rainfall(X) mm \& runoff(Y)mm } \\
\text { (Co.det.) }\end{array}$ & $\begin{array}{l}\text { Rainfall(X)mm \& soil loss(Z) t/ha } \\
\text { (Co.det) }\end{array}$ & $\begin{array}{l}\text { Runoff(Y)mm \& soil loss(Z)t/ha } \\
\text { (Co.det) }\end{array}$ \\
\hline TI - Rc Sole & $Y=0.36-0.01 I X+0.004 X 2(0.969)$ & $Z=0.03-0.006 X+0.0002 X 2(0.96)$ & $Z=-0.10 I+0.043 Y(0.976)$ \\
\hline T2- Pp Sole & $Y=0.49-0.015 X+0.004 X 2(0.966)$ & $Z=0.033-0.007 X+0.0002 \times 2(0.957)$ & $Z=-0.102+0.044 Y(0.983)$ \\
\hline T3-Gn Sole & $Y=0.426-0.004 X+0.004 X 2(0.968)$ & $Z=0.023-0.004 X+0.0002 \times 2(0.965)$ & $Z=-0.079+0.039 Y(0.988)$ \\
\hline $\begin{array}{l}\text { T4-Pp \& Rc strip } \\
\text { cropping }\end{array}$ & $Y=0.427-0.005 X+0.004 X 2(0.968)$ & $Z=0.023-0.004 X+0.0002 X 2(0.965)$ & $Z=-0.079+0.039 Y(0.988)$ \\
\hline $\begin{array}{l}\text { T5- Pp \& Gn strip } \\
\text { cropping }\end{array}$ & $Y=0.40 I-0.0003 X+0.004 X 2(0.969)$ & $Z=0.032-0.006 X+0.0002 X 2(0.958)$ & $Z=-0.1+0.043 Y(0.976)$ \\
\hline $\begin{array}{l}\text { T6-Rc+Pp }(5: 2) \\
\text { intercropping }\end{array}$ & $Y=0.383-0.002 X+0.004 X 2(0.969)$ & $Z=0.021-0.004 X+0.0002 \times 2(0.967)$ & $Z=-0.078+0.039 Y(0.988)$ \\
\hline $\begin{array}{l}\text { T7-Gn+Pp (4:2) } \\
\text { intercropping }\end{array}$ & $Y=0.346-0.008 X+0.004 X 2(0.970)$ & $Z=0.02-0.004 X+0.0002 \times 2(0.969)$ & $Z=-0.076+0.039 Y(0.987)$ \\
\hline $\begin{array}{l}\text { T8-Uncultivated } \\
\text { fallow }\end{array}$ & $Y=0.65-0.045 X+0.005 X 2(0.963)$ & $Z=0.04-0.008 X+0.0003 \times 2(0.952)$ & $Z=-0.104+0.044 Y(0.984)$ \\
\hline T9-Cultivated fallow & $Y=0.97-0.104 X+0.006 \times 2(0.955)$ & $Z=0.052-0.01 X+0.0003 \times 2(0.95 I)$ & $Z=-0.098+0.05 Y(0.996)$ \\
\hline
\end{tabular}

Table 8 Relation between Rainfall (X)mm, Runoff(Y)mm and Soil loss (Z) t/ha in different treatments along with co-efficient of determination (2008-09).

\begin{tabular}{|c|c|c|c|}
\hline \multirow{2}{*}{ Treatments } & \multicolumn{3}{|l|}{ Relations } \\
\hline & $\begin{array}{l}\text { Rainfall(X) mm \& runoff(Y)mm } \\
\text { (Co.det.) }\end{array}$ & $\begin{array}{l}\text { Rainfall(X)mm \& soil loss(Z) t/ha } \\
\text { (Co.det) }\end{array}$ & $\begin{array}{l}\text { Runoff(Y)mm \& soil loss(Z)t/ha } \\
\text { (Co.det) }\end{array}$ \\
\hline TI - Rc Sole & $Y=-0.5+0.156 X+0.002 X 2(0.975)$ & $Z=-0.019+0.003 X+0.0001 \times 2(0.973)$ & $Z=-0.075+0.041 Y(0.993)$ \\
\hline T2- Pp Sole & $Y=-0.432+0.152 X+0.002 \times 2(0.975)$ & $Z=-0.019+0.003 X+0.0001 \times 2(0.974)$ & $Z=-0.077+0.042 Y(0.994)$ \\
\hline T3-Gn Sole & $Y=-0.414+0.14 X+0.002 X 2(0.975)$ & $Z=-0.017+0.003 X+0.0001 \times 2(0.967)$ & $Z=-0.078+0.04 \mid Y(0.991)$ \\
\hline $\begin{array}{l}\text { T4-Pp \& Rc strip } \\
\text { cropping }\end{array}$ & $Y=-0.532+0.15 X+0.002 \times 2(0.977)$ & $Z=-0.01+0.002 X+0.0001 \times 2(0.976)$ & $Z=-0.075+0.042 Y(0.993)$ \\
\hline $\begin{array}{l}\text { T5- Pp \& Gn strip } \\
\text { cropping }\end{array}$ & $Y=-0.53 I+0.15 X+0.002 \times 2(0.977)$ & $Z=-0.018+0.003 X+0.0001 \times 2(0.976)$ & $Z=-0.075+0.042 Y(0.993)$ \\
\hline $\begin{array}{l}\text { T6-Rc+Pp (5:2) } \\
\text { intercropping }\end{array}$ & $Y=-0.57 I+0.15 X+0.002 X 2(0.974)$ & $Z=-0.018+0.003 X+0.0001 \times 2(0.973)$ & $Z=-0.074+0.042 Y(0.993)$ \\
\hline $\begin{array}{l}\text { T7-Gn+Pp (4:2) } \\
\text { intercropping }\end{array}$ & $Y=-0.513+0.136 X+0.002 X 2(0.972)$ & $Z=-0.018+0.003 X+0.0001 \times 2(0.961)$ & $Z=-0.08 I+0.04 I Y(0.99)$ \\
\hline $\begin{array}{l}\text { T8-Uncultivated } \\
\text { fallow }\end{array}$ & $Y=-0.1+0.14 X+0.004 X 2(0.976)$ & $Z=-0.018+0.003 X+0.0001 X 2(0.974)$ & $Z=-0.083+0.042 Y(0.996)$ \\
\hline T9-Cultivated fallow & $Y=-0.2+0.14 X+0.003 X 2(0.967)$ & $Z=-0.018+0.003 X+0.0001 \times 2(0.693)$ & $Z=-0.097+0.052 Y(0.997)$ \\
\hline
\end{tabular}


Table 9 Relation between Rainfall (X)mm, Runoff(Y)mm and Soil loss (Z) t/ha in different treatments along with co-efficient of determination (2009-I0)

\begin{tabular}{|c|c|c|c|}
\hline \multirow[b]{2}{*}{ Treatments } & \multicolumn{3}{|l|}{ Relations } \\
\hline & $\begin{array}{l}\text { Rainfall(X) mm \& runoff(Y)mm } \\
\text { (Co.det.) }\end{array}$ & $\begin{array}{l}\text { Rainfall(X)mm \& soil loss }(Z) t / h a \\
\text { (Co.det) }\end{array}$ & $\begin{array}{l}\text { Runoff(Y)mm \& soil loss(Z)t/ha } \\
\text { (Co.det) }\end{array}$ \\
\hline TI - Rc Sole & $Y=-I .33+0.384 X+0.00004 X 2(0.982)$ & $Z=-0.036+0.007 X+0.00004 X 2(0.985)$ & $Z=-0.082+0.031 Y(0.979)$ \\
\hline T2- Pp Sole & $Y=-1.35+0.387 X+0.00003 X 2(0.984)$ & $Z=-0.036+0.007 X+0.00004 \times 2(0.987)$ & $Z=-0.082+0.031 Y(0.98)$ \\
\hline T3-Gn Sole & $Y=-1.39+0.39 \mid X-0.00008 \times 2(0.98 I)$ & $Z=-0.037+0.007 X+0.00003 \times 2(0.984)$ & $Z=-0.085+0.031 Y(0.979)$ \\
\hline $\begin{array}{l}\text { T4-Pp \& Rc strip } \\
\text { cropping }\end{array}$ & $Y=-I .47+0.392 X-0.0002 X 2(0.973)$ & $Z=-0.41+0.007 X+0.00003 \times 2(0.975)$ & $Z=-0.092+0.031 Y(0.979)$ \\
\hline $\begin{array}{l}\text { T5- Pp \& Gn strip } \\
\text { cropping }\end{array}$ & $Y=-1.53+0.391 X-0.0002 X 2(0.975)$ & $Z=-0.041+0.007 X+0.00003 \times 2(0.978)$ & $Z=-0.075+0.03 Y(0.976)$ \\
\hline $\begin{array}{l}\text { T6-Rc+Pp }(5: 2) \\
\text { intercropping }\end{array}$ & $Y=-I .57+0.394 X-0.0004 X 2(0.974)$ & $Z=-0.045+0.008 X+0.000001 \times 2(0.907)$ & $Z=-0.04 I+0.024 Y(0.92 I)$ \\
\hline $\begin{array}{l}\text { T7-Gn+Pp (4:2) } \\
\text { intercropping }\end{array}$ & $Y=-I .59+0.388 X-0.0004 X 2(0.968)$ & $Z=-0.039+0.007 X+0.000002 X 2(0.900)$ & $Z=-0.046+0.024 Y(0.922)$ \\
\hline T8-Uncultivated fallow & $Y=-1.23+0.387 X+0.0003 X 2(0.983)$ & $Z=-0.042+0.009 X+0.00007 \times 2(0.985)$ & $Z=-0.127+0.041 Y(0.975)$ \\
\hline T9-Cultivated fallow & $Y=-1.23+0.4 \mid 4 X+0.0003 X 2(0.972)$ & $Z=-0.054+0.011 X+0.00006 \times 2(0.972)$ & $Z=-0.116+0.041 Y(0.986)$ \\
\hline
\end{tabular}

\section{Conclusion}

Thus it can be concluded that intercropping of groundnut with pigeon pea planted along contour may be practiced to increase crop yield and lowering the soil loss and runoff in the hilly tribal areas of Kandhamal district. The relationship among rainfall, runoff and soil loss was found out which can be used to predict the runoff and soil loss from rainfall for same type of soil condition and slope.

\section{Acknowledgement}

Author acknowledge the help of scientists of AICRPDA and staffs, Vice Chancellor, OUAT, Dean of Research, OUAT for time to time guidance. Author also acknowledge the help of P.C, AICRPDA, CRIDA, Hyderabad for all types of help.

\section{Conflict of interest}

None.

\section{References}

1. Samra JS. Watershed management a tool for sustainable production Proceedings of Indian Association of Soil \& Water Conservationists. Dehradun conference held in 2001. 2002;1-10.
2. Subudhi CR, PradhanPC, Senapati PC. Effect of grass bund on erosion loss and yield of rainfed rice, Orissa, India, T.Vetiver Network 1999;19:32-33.

3. Dinesh A, Gupta AK. Effect of water conservation measures in a pasture on the productivity of Buffel grass. Proceedings of Indian Association of Soil \& Water Conservationists. Dehradun conference held in 2001. 2002;65-66.

4. Munish Kumar. Impact of soil \& water conservation on erosion loss and yield of Kharif crops under ravenous watershed. Proceedings of Indian Association of Soil \& Water Conservationists. Dehradun conference held in 2001. 2002;301-303.

5. Annonymous. Annual progress report of AICRPDA. OUAT, Phulbani. 2006.

6. Eswaran VB. Wasteland development. Proceedings of Indian Association of Soil \& Water Conservationists. Dehradun conference held in 2001. 2002;17-19.

7. Patil PP. Soil and moisture conservation practices for the hill slopes in Western Ghat of Maharastra. Extended abstracts of National Conferences on Resource Conserving Technologies for Social Upliftment. 2004;122124. 